\title{
A FACILE SYNTHESIS OF 6,7-FUSED STEROIDAL THIAZOLES VIA 1,3-INTRAMOLECULAR MIGRATION OF BROMINE/ALLYLIC DISPLACEMENT OF BROMINE
}

\author{
MAHBOOB ALAM*广, SURAIYA KHAN AND MOHD. SHAHEEN KHAN \\ Department of Chemistry, Aligarh Muslim University, Aligarh 202002, India. \\ $\dagger$ Presently working as Guest-faculty \\ (Received: February 25, 2008 - Accepted: September 11, 2008)
}

\begin{abstract}
A facile synthesis of an array of 6,7-fused steroidal thiazoles (4-9) is reported. Replacement of lachrymatory 7-bromosteroidal ketones (4a) with readily accessible 5-bromosteroidal ketones (1-3), as a starting material, on simple condensation with thiourea or phenylthiourea gives the thiazole fused ring products. The products have been characterized on the basis of microanalytical and spectral data, which find support in some cases from comparison with previously known samples. A rationalization for the routes of conversion is given, based on current and previous results.
\end{abstract}

Keywords: Steroidal thiazoles, lachrymatory, phenylthiourea, IR spectroscopy.

\section{INTRODUCTION}

Thiazole is an important heterocycle found in various natural products, notably thiamine (vitamin B). ${ }^{1}$ Therefore, thiazole derivatives have been isolated and synthesized in view of their pharmacological activities viz antibacterial, antifungal, anti-inflammatory and antiviral..$^{2-6}$ These are also employed as antiallergic, ${ }^{7}$ anthelmintic ${ }^{8}$ and as sedative hypnotic agents. ${ }^{9}$ In addition, Nphenyl-1,3-thiazoles are expected to be important constituents of the medicines used for the treatment of multiple myeloma, chronic autoimmune disease, as well as AIDS. ${ }^{10}$ Due to the significance of thiazole derivatives in medicinal chemistry, considerable effort has been devoted to develop alternate routes for their synthesis. ${ }^{11-28}$

Although several strategies with variable yields have been reported for the synthesis of steroidal as well as non-steroidal thiazoles, the Hantzsch's protocol ${ }^{29-31}$ continues to be a method of choice involving the reaction of $\alpha$ halocarbonyl compound with an appropriate thiourea or thioamide. Thus, we have also selected the Hantzsch's protocol for this purpose, 5-bromosteroidal ketones, ${ }^{32-33}$ are selected instead of 7-bromosteroidal ketones because both lead to the same product but preparation of 7-bromocholestan-6-one ${ }^{20-21}$ is much more tedious leading to a mixture of products.

The paper describes the synthesis of thiazoles by simple condensation reaction between steroidal- $\alpha$-bromoketones and thiourea or phenylthiourea in methanol.

\section{EXPERIMENTAL}

Melting points were determined on a Kofler apparatus and are uncorrected. The compounds mentioned in this paper are known and their full characterization data, except ${ }^{13} \mathrm{C}$ NMR, have been previously reported in the literature. Confirmation of the compounds in our labs was done using, IR, ${ }^{1} \mathrm{H}$ NMR, ${ }^{13} \mathrm{C}$ NMR, MS and elemental analysis as appropriate. The IR spectra were recorded on $\mathrm{KBr}$ pellets with Pye Unicam SP3-100 spectrophotometer and its values are given in $\mathrm{cm}^{-1}$. Elemental analyses $(\mathrm{C}, \mathrm{H}$ and $\mathrm{N})$ were carried out with a Carlo Erba EA-1108 analyzer. ${ }^{1} \mathrm{H}-\mathrm{NMR}$ spectra were run in $\mathrm{CDCl}$, on a Bruker DRX-300 (300 MHz, FT-NMR) instrument with TMS as internal standard and its values are in ppm $(\delta) \cdot{ }^{13} \mathrm{C}$ NMR spectra were recorded on a Bruker Avance II 400 spectrometer in $\mathrm{CDCl}_{3}$. Mass Spectra were measured on JMS-D300, AIE MS-9 spectrometer using direct insertion technique at a source temperature of $250^{\circ} \mathrm{C}$. Thin layer chromatography (TLC) plates were coated with silica gel $\mathrm{G}$ and exposed to iodine vapors to check the purity as well as the progress of reaction. Petroleum benzine refers to a fraction of b.p. $60-80{ }^{\circ} \mathrm{C}$. Sodium sulfate (anhydrous) was used as a drying organic extracts after reaction work-up. All the solvents were distilled before use.

\section{Synthesis of steroidal thiazoles (Representing procedure)}

To a solution of 5-bromosteroidal ketone $(\mathbf{1}, \mathbf{2}$ or $\mathbf{3})(1 \mathrm{mmol})$ in methanol $(10 \mathrm{~mL})$ was added thiourea or phenylthiourea $(1 \mathrm{mmol})$ and the mixture was heated under reflux for $10 \mathrm{hrs}$. After completion of reaction, as determined by TLC, the reaction mixture was diluted with water and extracted thoroughly with ether. The extracts were washed successively with water and dried over anhydrous sodium sulfate $\left(\mathrm{Na}_{2} \mathrm{SO}_{4}\right)$, and concentrated to yield the steroidal thiazole $(4,5,6,7,8$ or 9$)$ as oil, which were crystallized from low fraction benzine (petroleum benzine) to obtain pure products. The results are given in table 1 and 2.

\section{RESULTS AND DISCUSSION}

The easily accessible steroidal 5-bromoketones (1, 2, and 3) were synthesized by a procedure described in the literature. ${ }^{32-35} \mathrm{~A}$ condensation reaction involving bromoketones and thiourea or phenylthiourea in methanol under reflux and subsequent processing leads to the formation of steroidal thiazoles having thiazole moiety at 6,7 position of ring B of cholestane skeleton. (4-9). The advantage of the reaction is to evade the lachrymatory 7 bromoketone (4a), as a starting material. The striking feature of the reaction is either 1,3-intramolecular shift of bromine ${ }^{32}$ or allylic displacement of bromine during the formation of desired products. The reaction of $3 \beta$-acetoxy-5-bromo-

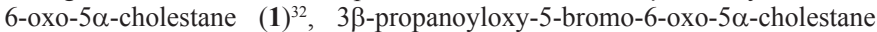
$(2)^{33}$ and 5-bromo-6-oxo-5 $\alpha$-cholestane (3), ${ }^{34-35}$ with thiourea or phenylthiourea in methanol under reflux for about 8-10 hours afforded $3 \beta$-acetoxy-2'-amino$5 \alpha$-cholestan-6-eno[6,7- $d$ ] thiazole (4) ${ }^{20-21}$ or $3 \beta$-acetoxy-2'-N-phenylamino$5 \alpha$-cholestan-6-eno $[6,7-d]$ thiazole $(7)$ and its series compounds $(\mathbf{5}, \mathbf{8})$ and $(\mathbf{6}$, 9), respectively, (Scheme 1), in moderate yields.

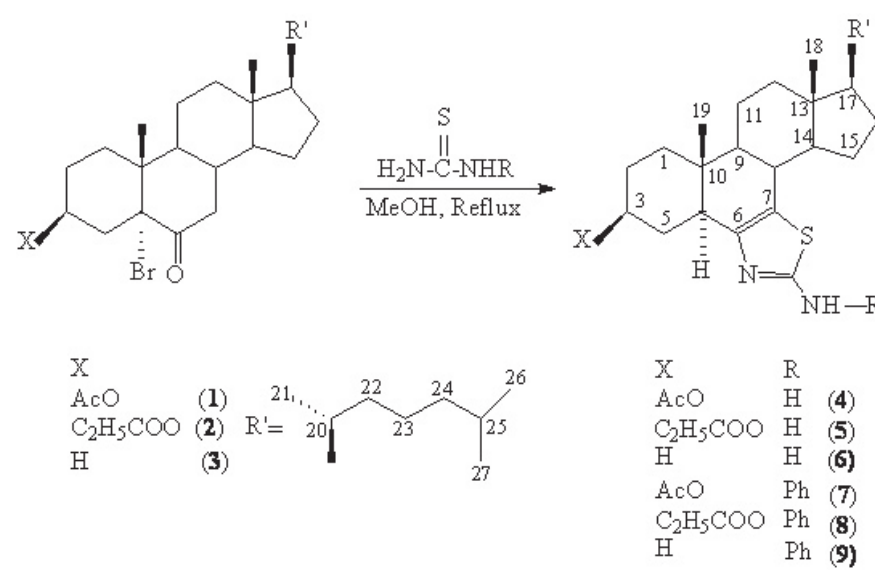

Scheme1. Synthesis of fused steroidal thiazoles

The structure of compound (4) was established by IR, ${ }^{1} \mathrm{H}$ NMR, ${ }^{13} \mathrm{C}$ NMR, MS and microanalyical data. Compound (4) gave diagnostic IR bands at 3240 $\mathrm{cm}^{-1}\left(-\mathrm{NH}_{2}\right), 1730$ and $1265 \mathrm{~cm}^{-1}\left(\mathrm{CH}_{3} \mathrm{COO}-\right), 1630 \mathrm{~cm}^{-1}(\mathrm{C}=\mathrm{C})$ and $1540 \mathrm{~cm}^{-1}$ $(\mathrm{C}=\mathrm{N}){ }^{36}$ Some other bands were also observed at $1320 \mathrm{~cm}^{-1}$ for $(\mathrm{C}-\mathrm{N})$ and 665 $\mathrm{cm}^{-1}$ for $(\mathrm{C}-\mathrm{S})^{36}$ stretching frequencies supporting the presence of the thiazole moiety. The ${ }^{1} \mathrm{H}$ NMR spectrum exhibited a broad singlet at $\delta 5.31$ integrating for two protons corresponding to the $-\mathrm{N}_{2}$ group and another broad peak appeared at $\delta 4.93$ for $\mathrm{C}_{5}-\alpha \mathrm{H}$. A multiplet was observed, integrating for one proton at $\delta$ $4.71\left(\mathrm{~W}_{12}=17 \mathrm{~Hz}\right)$, which could be attributed to the $\mathrm{C} 3-\alpha \mathrm{H}$, axial proton $(\mathrm{A} / \mathrm{B}$ ring junction trans). ${ }^{37}$ The methyl protons of the acetate group appeared at $\delta 2.10$ as a sharp singlet. However, the angular methyl protons $\left(\mathrm{C}_{10}-\mathrm{CH}_{3}\right),\left(\mathrm{C}_{13}-\mathrm{CH}_{13}\right)$ as siglets and side chain methyl protons $\left(\mathrm{C}_{21}-\mathrm{CH}_{3}\right),\left(\mathrm{C}_{25}-\left(\mathrm{CH}_{3}\right)_{2}\right)$ as doublets, were observed at $\delta 1.21,0.92,0.81$ and 0.67 respectively. In the ${ }^{13} \mathrm{C}$ NMR spectrum, the peak appearing in the range of $\delta 162-166 \mathrm{ppm}$ corresponds to C-2' of the thiazole ring and other characteristic ${ }^{13} \mathrm{C}$ NMR signals appeared at $\delta 170.67$ 
$\left(\mathrm{CH}_{3} \mathrm{COO}\right), 149.01(\mathrm{C}-6)$ and 122.75 (C-7) respectively (Fig. 2). The absence of a carbonyl as well as an $\alpha$-bromo group absorption at $1710(\mathrm{C}=\mathrm{O})$ and 780 (C...Br) $\mathrm{cm}^{-1}$ confirms the formation of (4). Thus, the above data supports the formation of $3 \beta$-acetoxy-2'-amino-5 $\alpha$-cholestan-6-eno $[6,7-d]$ thiazole (4). The identity of $\mathbf{4}$, was further supported by m.p., mixed m.p., (reported, m.p., $202{ }^{\circ} \mathrm{C}$ ) and co-tlc..$^{20-21}$ The products $(\mathbf{5 - 9})$ were also characterized on the basis of similar accounts. Besides the ${ }^{13} \mathrm{C}$ NMR data (The structure elucidation of 4-9 is mainly based on ${ }^{13} \mathrm{C}$ NMR spectroscopy see Experimental for details) and elemental analysis, the appearance of the molecular ion peak at $\mathrm{m} / \mathrm{z} 500$ $\left(\mathrm{M}^{+}\right)$is in good agreement with the molecular formula $\mathrm{C}_{30} \mathrm{H}_{48} \mathrm{~N}_{2} \mathrm{O}_{2} \mathrm{~S}(4)$ which further establishes its formation (Fig. 1). Ahmad ${ }^{38}$ has reported a similar set of title compounds starting from the same material but with a different solvent, methanol but provided no satisfactory mechanism. However, Shamsuzzaman et al. ${ }^{39}$ reported different steroidal thiazoles starting from similar precursor using ethanol as a solvent with a refluxing period of 2-3 hrs.

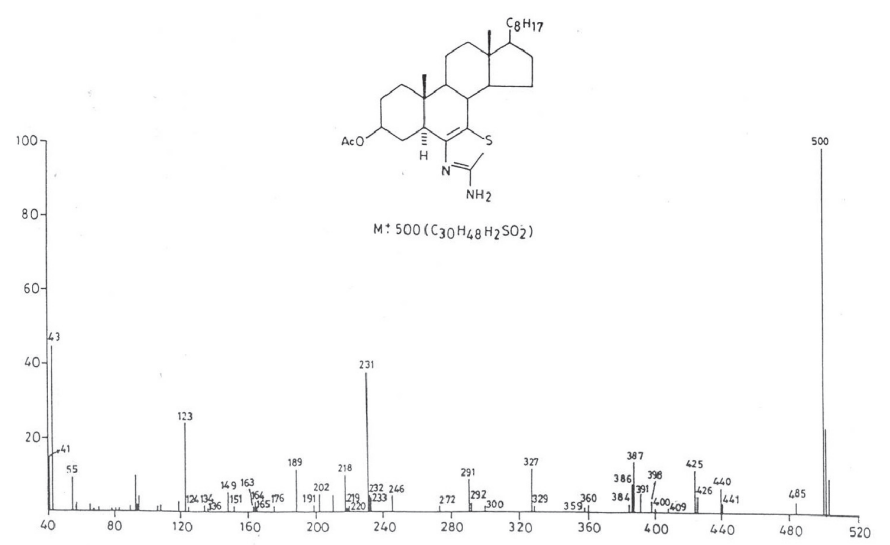

Fig. 1 Fragmentation pattern of compound 4

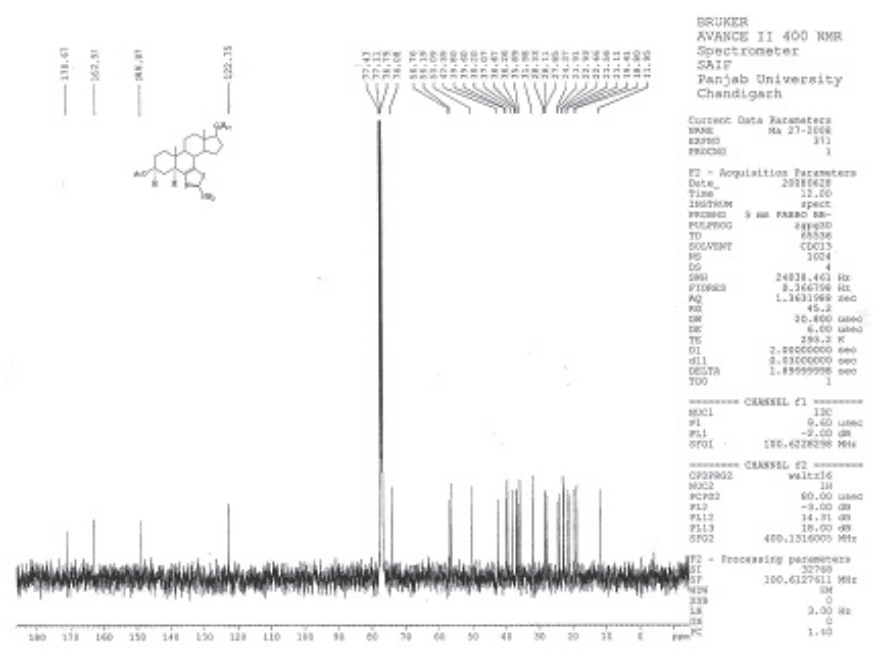

Fig. 2. ${ }^{13} \mathrm{C}$ NMR spectrum of compound 4 in $\mathrm{CDCl}_{3}$

The effect of solvent in synthesis of thiazoles could not be ruled out. The study of solvent effects as well as reaction conditions ${ }^{40}$ in the synthesis of thiazoles is in progress for future reports. The formation of product (4) can be explained considering that during the reaction, 1,3 shift of bromine ${ }^{32}$ from $\mathrm{C} 5$ to C7 leads to the formation of intermediate 4a in situ (scheme 3), followed by $\mathrm{S}_{\mathrm{N}}{ }^{2}$ attack of sulfur of the reagent and subsequent cyclization, which gives the product (4). The formation of steroidal thiazole could also be explained by an alternate route, considering that during the reaction, allylic displacement of bromine and subsequent attack of sulfur atom of reagent followed by cyclization, leading to the formation of the desired product (4). It is expected that driving force to accelerate reaction towards product direction is an enol tautomeric form as established by ${ }^{1} \mathrm{H}$ NMR spectroscopy in similar case of thaizoles. ${ }^{41}$ The tentative mechanism, explaining the observed products (59), has been proposed on the basis of spectral studies as well as current and previous results. $^{42}$

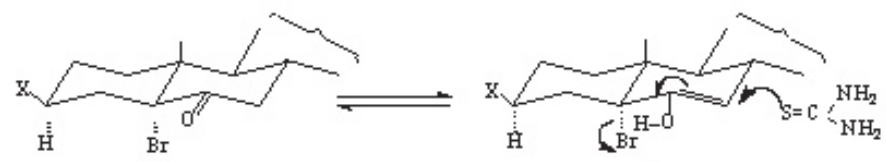

(1)
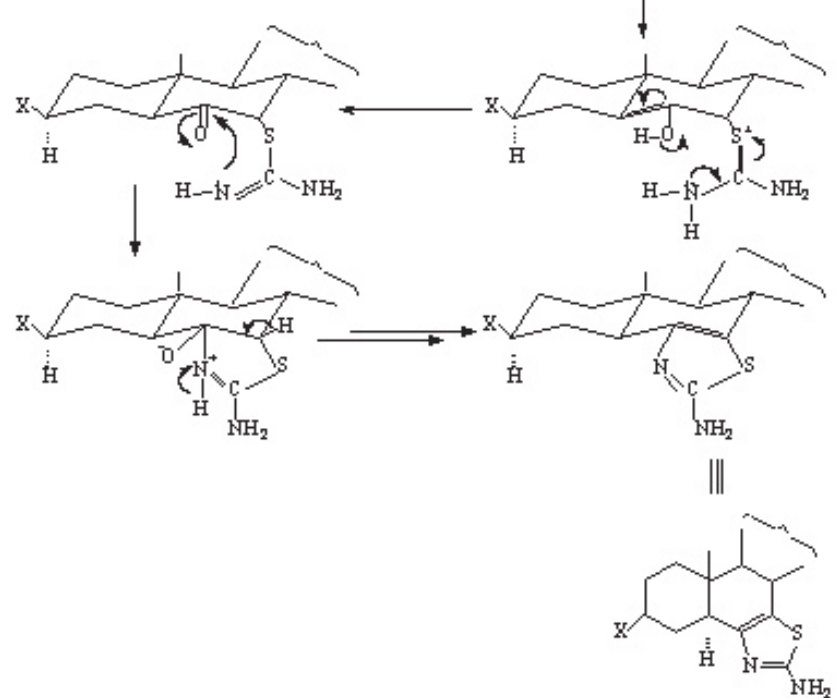

(4)

Schem e-2. Allylic displac em ert of brom ine by the attack of sulfir atom of re agert
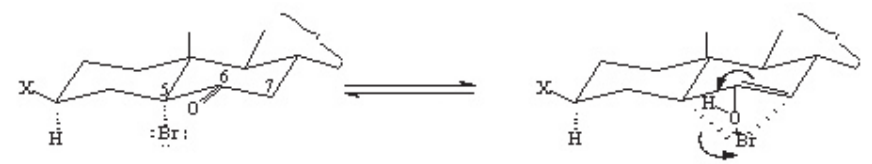

(1)

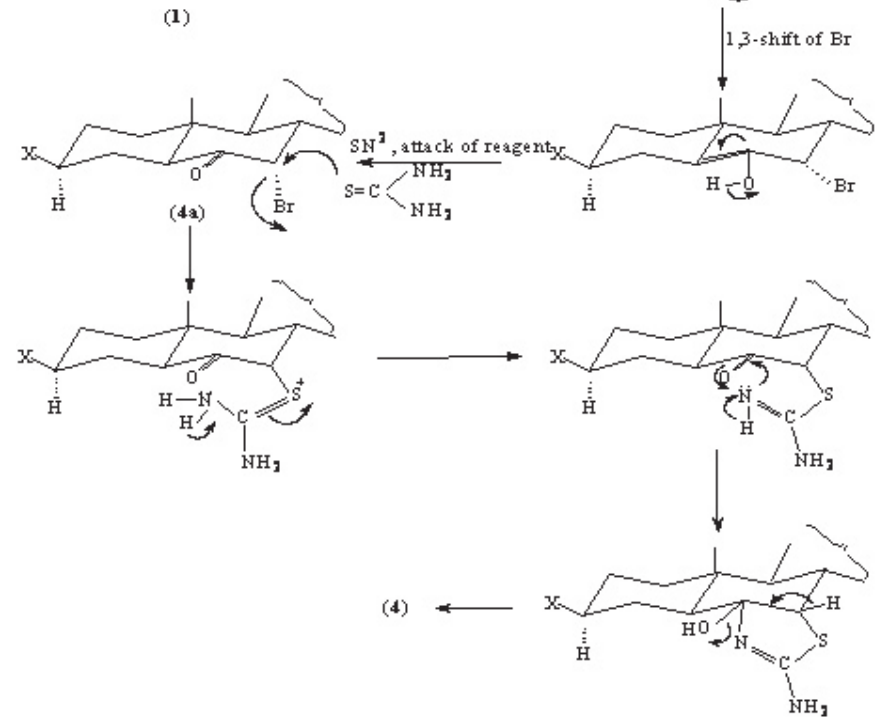

3-shift of $\mathrm{Br}$

Scheme-3.13-shift of bromine followed by $\mathrm{SH}^{\mathbf{3}}$ attack of Sulfur atom of reszent 


\section{CONCLUSION}

The present work describes a facile synthesis of steroidal thiazoles starting from conveniently accessible precursor 5-bromosteroidal ketones instead of lachrymatory 7-bromosteroidal ketones. The reported compounds can serve as biologically relevant compounds as their analogs were found to possess versatile anti-inflammatory and anti-tuberculosis activities.

Table 1. Characterization data of the compounds (4 to 9).

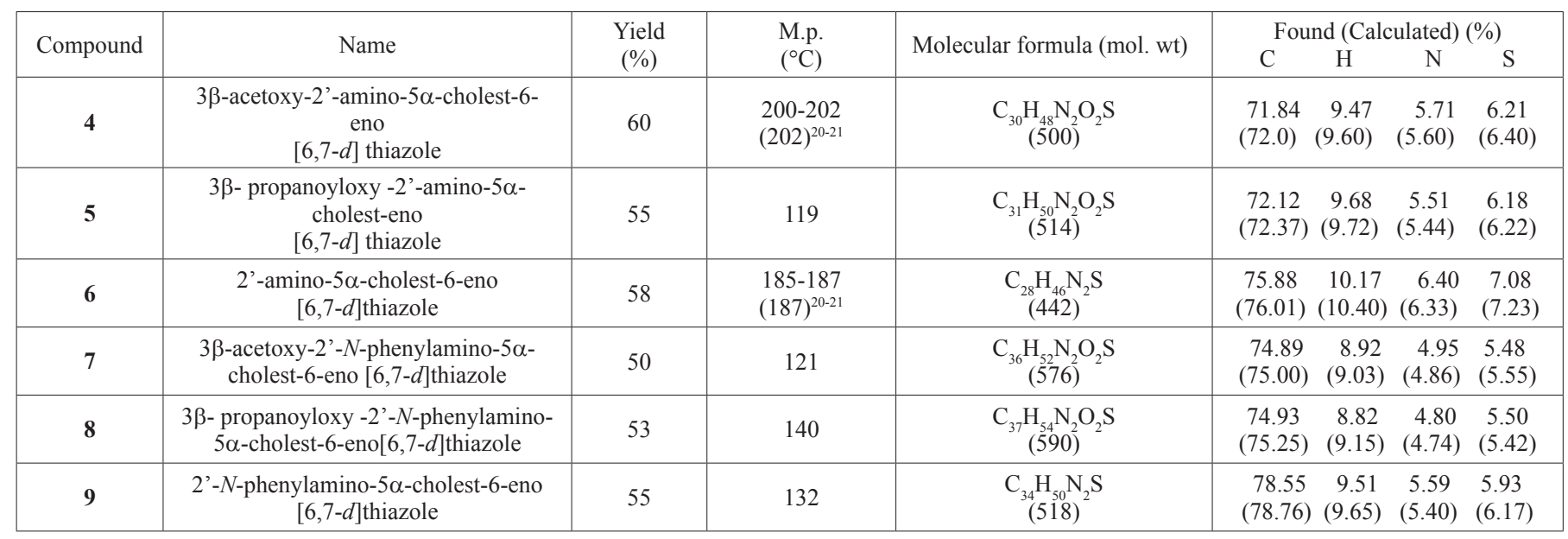

Table 2. Spectral data of the compounds (4 to 9)

\begin{tabular}{|c|c|c|c|}
\hline Compound & $\operatorname{IR}(\mathrm{KBr}) \mathrm{cm}^{-1}$ & ${ }^{1} \mathrm{HNMR}\left(\mathrm{CDCl}_{3}\right)(\delta, \mathrm{ppm})$ & MS: m/z \\
\hline 4 & $\begin{array}{l}3240\left(-\mathrm{NH}_{2}\right), 1730,1265 \\
\left(\mathrm{CH}_{3} \mathrm{COO}\right), 1630(\mathrm{C}=\mathrm{C}), 540 \\
(\mathrm{C}=\mathrm{N}), 1320(\mathrm{C}-\mathrm{N}) \text { and } 650 \\
(\mathrm{C}-\mathrm{S})\end{array}$ & $\begin{array}{l}5.3 \text { (brs, } 2 \mathrm{H},-\mathrm{NH}_{2} \text { exchangeable with deuterium), } 4.93 \\
\left.\text { (brs, } 1 \mathrm{H}, \mathrm{C}_{5}-\alpha \mathrm{H}\right), 4.71\left(\mathrm{~m}, 1 \mathrm{H}, \mathrm{W}_{1 / 2}=18 \mathrm{~Hz}, \mathrm{C}_{3 \alpha}-\mathrm{H}\right) \\
2.05\left(\mathrm{~s}, 3 \mathrm{H}_{1}, \mathrm{CH}_{3} \mathrm{COO}^{-}\right), 1.21\left(\mathrm{~s}, 3 \mathrm{H}_{1}, \mathrm{C}_{10}-\mathrm{CH}_{3}\right), 0.67 \\
\left.\text { (s, 3H, } \mathrm{C}_{13}-\mathrm{CH}_{3}\right), 0.92,0.81 \text { (other side chain methyl } \\
\text { protons). }\end{array}$ & $\begin{array}{l}500\left(\mathrm{M}^{+}\right), 485\left(\mathrm{M}^{+}-\mathrm{CH}_{3}\right), 387\left(\mathrm{M}^{+}-\mathrm{C}_{8} \mathrm{H}_{17}\right), \\
440\left(\mathrm{M}^{+}-\mathrm{CH}_{3} \mathrm{COOH}\right), 425\left(\mathrm{M}^{+}-\mathrm{CH}_{3} \mathrm{COOH}\right. \\
\left.\text { and } \mathrm{CH}_{3}\right), 373\left(\mathrm{M}^{+}-\mathrm{CH}_{3} \mathrm{COOH}, \mathrm{CH}_{3} \text { and }\right. \\
\left.\mathrm{CH}_{2} \mathrm{~N}_{2}\right), 398\left(\mathrm{M}^{+}-\mathrm{CH}_{3} \mathrm{COOH} \text { and } \mathrm{CH}_{2} \mathrm{~N}_{2}\right) \\
\text { and } 327\left(\mathrm{M}^{+}-\mathrm{CH}_{3} \mathrm{COOH} \text { and } \mathrm{C}_{8} \mathrm{H}_{17}\right) .\end{array}$ \\
\hline 5 & $\begin{array}{l}3200\left(-\mathrm{NH}_{2}\right), 1735,1240 \\
\left(\mathrm{CH}_{3} \mathrm{CH}_{2} \mathrm{COO}\right), 1520(\mathrm{C}=\mathrm{N}) \\
1450(\mathrm{C}-\mathrm{N}) \text { and } 660(\mathrm{C}-\mathrm{S})\end{array}$ & $\begin{array}{l}5.2 \text { (brs, } 2 \mathrm{H},-\mathrm{NH}_{2} \text { exchangeable with deuterium) }, 4.81 \\
\left.\text { (brs, } 1 \mathrm{H}, \mathrm{C}_{5}-\mathrm{\alpha H}\right), 4.9\left(\mathrm{~m}, 1 \mathrm{H}, \mathrm{C}_{3 \alpha}-\mathrm{H}, \mathrm{W}_{1 / 2}=18 \mathrm{~Hz}\right), 2.2 \\
\left(\mathrm{q}, 2 \mathrm{H}, \mathrm{CH}_{3} \mathrm{CH}_{2} \mathrm{COO}, \mathrm{J}=7 \mathrm{~Hz}\right), 1.21\left(\mathrm{~s}, 3 \mathrm{H}, \mathrm{C}_{10}-\mathrm{H}_{3}\right) \\
\left.0.69 \text { (s, } 3 \mathrm{H}_{1} \mathrm{C}_{13}-\mathrm{CH}_{3}\right), 0.92,0.81 \text { (other side ethyl } \\
\text { protons). }\end{array}$ & $\begin{array}{l}574\left(\mathrm{M}^{+}\right), 499\left(\mathrm{M}^{+}-\mathrm{CH}_{3}\right), 401\left(\mathrm{M}^{+}-\right. \\
\left.\mathrm{C}_{8} \mathrm{H}_{17}\right), 440\left(\mathrm{M}^{+}-\mathrm{CH}_{3} \mathrm{CH} / \mathrm{COOH}\right), \\
327\left(\mathrm{M}^{+}-\mathrm{CH}_{3} \mathrm{CH}_{2} \mathrm{COOH} \text { and } \mathrm{C}_{8} \mathrm{H}_{17}\right), \\
300\left(\mathrm{M}^{+}-\mathrm{CH}_{3} \mathrm{CH}_{2} \mathrm{COOH} \text { and } \mathrm{C}_{10} \mathrm{H}_{20}\right) \text {, } \\
386\left(\mathrm{M}^{+}-\mathrm{CH}_{3} \mathrm{CH}_{2} \mathrm{COOH} \text { and } \mathrm{C}_{4} \mathrm{H}_{6}\right), 273 \\
\left(\mathrm{M}^{+}-\mathrm{CH}_{3} \mathrm{CH}_{2} \mathrm{COOH}_{4} \mathrm{C}_{4} \mathrm{H}_{6} \text { and } \mathrm{C}_{8} \mathrm{H}_{17}\right) \text { and } \\
231\left(\mathrm{M}^{+}-\mathrm{CH}_{3} \mathrm{CH}_{2} \mathrm{CO} \mathrm{OH}, \mathrm{C}_{4} \mathrm{H}_{6}, \mathrm{C}_{8} \mathrm{H}_{17} \text { and }\right. \\
\left.-\mathrm{NCNH}_{2}\right) .\end{array}$ \\
\hline 6 & $\begin{array}{l}3250,3150\left(-\mathrm{NH}_{2}\right), 1625 \\
(\mathrm{C}=\mathrm{C}), 1550(\mathrm{C}=\mathrm{N}), 1470,1370 \\
(\mathrm{C}-\mathrm{N}) \text { and } 665(\mathrm{C}-\mathrm{S})^{35}\end{array}$ & $\begin{array}{l}5.3 \text { (brs, } 2 \mathrm{H},-\mathrm{NH}_{2} \text { exchangeable with deuterium), } 1.0 \\
0.92,0.81 \text { and } 0.65 \text { (other side chain methyl protons). }\end{array}$ & $\begin{array}{l}442\left(\mathrm{M}^{+}\right), 427\left(\mathrm{M}^{+}-\mathrm{CH}_{3}\right) 400\left(\mathrm{M}^{+}-\mathrm{CH}_{2} \mathrm{~N}_{2}\right) \\
386\left(\mathrm{M}^{+}-\mathrm{C}_{4} \mathrm{H}_{8}\right), 329\left(\mathrm{M}^{+}-\mathrm{C}_{8} \mathrm{H}_{17}\right), 302\left(\mathrm{M}^{+}-\right. \\
\left.\mathrm{C}_{10} \mathrm{H}_{20}\right) \text { and } 301\left(\mathrm{M}^{+}-\mathrm{H}\right) .\end{array}$ \\
\hline 7 & $\begin{array}{l}3000,1600(\text { aromatic gp) } \\
3200,3150(-\mathrm{NH}), 1735,1235 \\
\left(\mathrm{CH}_{3} \mathrm{COO}\right), 1520(\mathrm{C}=\mathrm{N}) \text { and } \\
650(\mathrm{C}-\mathrm{S}) .\end{array}$ & $\begin{array}{l}\text { 7.4, } 7.3(5 \mathrm{H}, \text { aromatic protons), } 6.5 \text { (brs, } \mathrm{s}, 1 \mathrm{H},-\mathrm{NH}) \\
3.8\left(\mathrm{~m}, 1 \mathrm{H}, \mathrm{C}_{3 \alpha}-\mathrm{H}, \mathrm{W}_{1 / 2}=16 \mathrm{~Hz}\right), 2.0\left(\mathrm{CH}_{3} \mathrm{COO}\right), 1.02, \\
0.91,0.81 \text { and } 0.70 \text { (others methyl protons). }\end{array}$ & $\begin{array}{l}576\left(\mathrm{M}^{+}\right), 575\left(\mathrm{M}^{+}-\mathrm{H}\right), 484\left(\mathrm{M}^{+}-\mathrm{NHPh}\right), \\
499\left(\mathrm{M}^{+}-\mathrm{Ph}\right), 359\left(\mathrm{M}^{+}-\mathrm{C}_{8} \mathrm{H}_{17} \mathrm{CH}=\mathrm{CH}_{2}\right), \\
561\left(\mathrm{M}^{+}-\mathrm{CH}_{3}\right), 463\left(\mathrm{M}^{+}-\mathrm{C}_{8} \mathrm{H}_{17}\right), 436\left(\mathrm{M}^{+}-\right. \\
\left.\mathrm{C}_{10} \mathrm{H}_{20}\right) \text { and } 516\left(\mathrm{M}^{+}-\mathrm{CH}_{3} \mathrm{CO} \mathrm{OH}\right) .\end{array}$ \\
\hline 8 & $\begin{array}{l}3000,1600(\text { aromatic gp), } \\
3200(-\mathrm{NH}), 1735,1210 \\
\left(\mathrm{CH}_{3} \mathrm{CH}_{2} \mathrm{COO}\right), 1520(\mathrm{C}=\mathrm{N}) \\
\text { and } 660(\mathrm{C}-\mathrm{S})\end{array}$ & $\begin{array}{l}\text { 7.4, } 7.3\left(\mathrm{~m}, 5 \mathrm{H} \text { aromatic protons), } 4.9\left(\mathrm{~m}, 1 \mathrm{H}, \mathrm{C}_{3 \alpha}-\mathrm{H} \text {, }\right.\right. \\
\left.\mathrm{W}_{1 / 2}=18 \mathrm{~Hz}\right), 6.1(\mathrm{brs}, 1 \mathrm{H},-\mathrm{NH}), 2.3\left(\mathrm{q}, \mathrm{CH}_{3} \mathrm{C}_{2} \mathrm{COO} \text {, }\right. \\
\mathrm{J}=7 \mathrm{~Hz}), 1.30,1.18,0.96 \text { and } 0.68 \text { (other methyl } \\
\text { protons). }\end{array}$ & $\begin{array}{l}590\left(\mathrm{M}^{+}\right), 477\left(\mathrm{M}^{+}-\mathrm{C}_{8} \mathrm{H}_{17}\right), 575\left(\mathrm{M}^{+}-\mathrm{CH}_{3}\right) \\
589\left(\mathrm{M}^{+}-\mathrm{H}\right), 498\left(\mathrm{M}^{+}-\mathrm{NHPh}\right), 516\left(\mathrm{M}^{+}-\right. \\
\left.\mathrm{CH}_{3} \mathrm{CH}_{2} \mathrm{COOH}\right) \text { and } 450\left(\mathrm{M}^{+}-\mathrm{C}_{10} \mathrm{H}_{20}\right)\end{array}$ \\
\hline 9 & $\begin{array}{l}\text { 3010, } 1600(\text { aromatic gp), } 3240 \\
(-\mathrm{NH}), 1530(\mathrm{C}=\mathrm{N}), 1320(\mathrm{C}-\mathrm{N}) \\
\text { and } 600(\mathrm{C}-\mathrm{S})\end{array}$ & $\begin{array}{l}\text { 7.4, } 7.3 \text { (m, } 5 \mathrm{H} \text {, aromatic protons), } 6.6 \text { (brs, } 1 \mathrm{H},-\mathrm{NH} \\
\text { exchangeable with deuterium), } 1.12,0.91,0.85 \text {, and } \\
0.65 \text { (other methyl protons). }\end{array}$ & $\begin{array}{l}518\left(\mathrm{M}^{+}\right), 517\left(\mathrm{M}^{+}-\mathrm{H}\right), 503\left(\mathrm{M}^{+}-\mathrm{CH}_{3}\right), \\
462\left(\mathrm{M}^{+}-\mathrm{C}_{4} \mathrm{H}_{8}\right), 461\left(\mathrm{M}^{+}-\mathrm{C}_{4} \mathrm{H}_{8} \text { and } \mathrm{H}\right), 441 \\
\left(\mathrm{M}^{+}-\mathrm{Ph}\right), 426\left(\mathrm{M}^{+}-\mathrm{NHPh}\right), 411\left(\mathrm{M}^{+}-\mathrm{NHPH}\right. \\
\left.\text { and } \mathrm{CH}_{3}\right) \text { and } 503\left(\mathrm{M}^{+}-\mathrm{CH}_{3}\right) .\end{array}$ \\
\hline
\end{tabular}

${ }^{13} \mathrm{C}$ NMR $\left(\mathrm{CDCl}_{3}\right)(\delta, \mathrm{ppm})(4) ; 170.67$ ( $\left.\mathrm{CH}_{3} \mathrm{COO}-\right), 162.91$ (C-2'), 149.01 (C-6), 122.75 (C-7), 74.08 (C-3), 56.76 (C-14), 56.19 (C-17), 50.09 (C-9), 42.93 (C-13), 39.80 (C-5), 39.60 (C-12), 38.20 (C-8), 37.07 (C-1), 36.67 (C-20), 36.26 (C-22), 35.89 (C-10), 31.98 (C-4), 28.33 (C-16), 28.11 (C-24), 27.85 (C-2)*, 24.37 (C-23), 23. 91 (C-15), 22.93 (C-25)*, 22.66 (C-11/C-27), 21.56 (C-19), 21.11 (CH COO-), 19.41 (C-26), 18.80 (C-21) and 11.95 (C-18), Asterisks denote assignments that may be interchanged; (5); 170.69 ( $\mathrm{CH}_{3} \mathrm{CH}_{2}$ COO-), $164.91\left(\mathrm{C}-2\right.$ '), 149.61 (C-6), $123.25(\mathrm{C}-7), 73.18$ (C-3), $27.71,9.21\left(\underline{\mathrm{C}} \mathrm{H}_{3} \mathrm{CH}_{2} \mathrm{COO}-\right),(6)$; 163.50 (C-2'), 149.35 (C-6), 122.35 (C-7), $33.50(\mathrm{C}-1), 20.61^{2}(\mathrm{C}-2)^{*}, 20.78(\mathrm{C}-3)^{*}, 33.17(\mathrm{C}-4), 47.18(\mathrm{C}-5) ;(7) ; 170.67\left(\mathrm{CH}_{3} \mathrm{COO}-\right), 165.91\left(\mathrm{C}-2^{3}\right), 149.41(\mathrm{C}-6)$, 140.75, 128.99, 124.66, 121.89 (aromatic gp), 122.89 (C-7), 74.07 (C-3), $21.11\left(\mathrm{CH}_{3} \mathrm{COO}-\right),(8) ; 170.67$ (CH $\left.\mathrm{COO}^{-}\right), 165.91$ (C-2'), 149.41 (C-6), $140.75,128.99$, 124.66, 121.89 (aromatic gp), 122.89 (C-7), 74.07 (C-3), 21.11 ( CH $\left._{3} \mathrm{COO}-\right),(9), 162.89$ (C-2'), 149.31 (C-6), 138.82, 128.98, $121.89,117.91$ (aromatic gp), 123.01 (C-7), 20.61 (C-2), 20.78 (C-3), 33.17 (C-4), 47.18 (C-5) and for other signals ( $\left.{ }^{13} \mathrm{CNMR}\right)$ are in close accord with the cholestane series. 


\section{ACKNOWLEDGEMENT}

We thank the Chairman, Department of Chemistry, A.M.U., Aligarh, for providing necessary research facilities. We wish to thank Professor M. Mushfiq for helpful discussions and his interest in our work. We are grateful to the SAIF, Panjab University for providing IR, ${ }^{1} \mathrm{H}$ NMR, ${ }^{13} \mathrm{C}$ NMR and elemental analysis

\section{REFERENCES}

1. J. Jensen, N. Skajaerabaek, Pervedso, Synthesis 128, (2001).

2. A. R. Katritzky, S. Strah, S. A. Belyakov, Tetrahedron 54, 7167, (1998), and references cited therein.

3. F. Russo, M. Santagati, Farm. Ed. Sci. 31, 41, (1976).

4. Y. Usui, Ann. Rep. Takeda Res. Lab. (Jpn), 27, 144, (1968).

5. S. C. Kuo, L. J. Huang, H. Nakamura, J. Med. Chem. 27, 539, (1984).

6. P. Schauer, M. Lkar, B. Stanovnik, M. Fisler, Biol. Vestn. 20, 65, (1972).

7. J. H. Musser, R. E. Brown, B. Love, K. Kaily, H. Jones, R. Kahen, F. Huang, A. Khandwala, M. Leibowetz, J. Med. Chem. 27, 121, (1984).

8. H. D. Brown, US. Pat. 3278 547, (1966); Chem. Abstr. 65, 18593, (1966).

9. Y. Sawa, T. Ishida, J. Pharm. Soc. Jpn. 76, 337, (1956).

10. K. Yamaguchi, M. Yado, T. Tsuji, Y. Hatanaka, K. Goda, T. Kobori, Bioorg. Med. Chem. Lett. 9, 957, (1999).

11. R. S. Verma, Pure App. Chem. 73, 193, (2001).

12. M. M. V. Raman, D. S. Dubhashi, J. J. D’ Souza, J. Chem. Res. (S), 496, (1998).

13. R. M. Moriarty, B. K. Vaid, M. P. Duncan, S. G. Levy, O. Prakash, S. Goyal, Synthesis 845, (1992).

14. J. Habermann, S. V. Ley, J. J. Scicinski, J. S. Scott, R. Smits, A. W. Thomson, J. Chem. Soc. Perkin Trans. 1, 2425, (1999).

15. K. C. Nicolaou, P. S. Baron, Y. L. Zhong, J. Am. Chem. Soc. 122, 10246, (2000).

16. B. Henkel, B. Beck, B. Westner, B. Mejat, A. Domling, Tetrahedron Lett. 44 8947, (2003).

17. R. S. Varma, D. Kumar, P. J. Liesen, J. Chem. Soc. Perkin Trans. 14093 , (1998).

18. G. K. Sarmah, N. K. Bhattacharyya, P. Goswomi, Barua, J. C. S. Kataky, J. Indian Chem. Soc. 80, 1163, (2003).
19. A. Hugershoff, Ber. 36, 3212, (1908).

20. Shafiullah, I. H. Siddiqui, S. Ahmad, Ind. J. Chem. 27B, 279, (1998).

21. Shafiullah, I. H. Siddiqui, Acta. Chimica Hungarica 1, 126, (1989).

22. J. C. Meslin, H. Quiniou, Tetrahedron 31, 3055, (1975).

23. S. Rajappa, Heterocycles 7, 507, (1977).

24. A. Reliquent, J. Meslin, F. Reliquent, Sulfur Lett. 7, 49, (1978).

25. A. M. Farag, K. M. Dawood, Z. E. Kandeel, M. S. Algharib, J. Chem. Res. (S), 530, (1996).

26. B. Kalluraya, P. Gunaga, M. V. Raman, Ind. Heterocycl. Chem. 241, (1998).

27. V. V. Doblatyan, K. A. Eliazan, V. A. Pivazyan, E. A. Ghazaryan, A. P. Engoyan, R. T. Grigoryan, R. G. Mirzoyan, Chem. Heterocycl. Compd. (N.Y), 36, 593, (2003).

28. M. D. Brown, D. W. Gillon, G. D. Meakins, G. A. Whithan, J. Chem. Soc. Chem. Commun. 444, (1982).

29. A. Hantzsch, H. Weber, Ber. Dtsch. Chem. Ges. 20, 3118, (1887).

30. V. Traumann, Justus Liebigs Ann. Chem. 249, (1888).

31. M. Mushfiq, A. Mahboob, J. Chin. Chem. Soc. 54, 219, (2007).

32. I. M. Heliborn, E. R. H. Jones, F. S. Spring, J. Chem. Soc. 801, (1937).

33. M. S. Ahmad, S. Z. Ahmad, I. A. Ansari, Ind. J. Chem. 25B 1161, (1986); S. Z. Ahmad, Ph.D. Thesis A.M.U., Aligarh, India, 1986.

34. C. W. Shoppe, R. H. Jewlens, G. H. R. Summers, J. Chem. Soc. 2492, (1956).

35. H. B. Henbest, T. I. Wringly, J. Chem. Soc. 4596, (1957).

36. L. J. Bellamy The Infrared Spectra of Complex Molecules, Methuen \& Co. Ltd., London, 1958.

37. N. S. Bhacca and D. H. Williams Application of NMR spectroscopy in organic chemistry, Holden Day, San Fransisco, 1964.

38. D. Ahmad, Ph.D. Thesis A.M.U. Aligarh, India, 1992.

39. Shamsuzzaman, A. Salim, M. K. Akram, K. Saleem, N. Siddiqui, Ind. J. Chem. 42B, 2872, (2003).

40. B. W. Lee, S. D. Lee, Tetrahedron Lett. 41, 3883, (2000).

C. M. Yang-i Lin, S. M. Seifert, J. P. Kang, S. A. Lang, J. Heterocycl. Chem. 16 1377, (1979); P. T. Kaye, G. D. Meakins, A. K. Smith, M. D. Tirel, J. Chem. Soc. Perkin Trans. 1, 1677, (1983); J. C. Meslin, H. Quiniou, Tetrahedron 31, 3055, (1975); J. C. Meslin, H. Quiniou, Synthesis 298, (1974). 